\title{
A Metamaterial-Based Microwave Sensor for Liquid Dielectrics Characterization
}

\author{
Chemseddine Zebiri ${ }^{1,2}$, Widad Mshwat ${ }^{2}$, Jamal Kosha ${ }^{2}$, Mohamed Lashab ${ }^{3}$, Issa Elfergani ${ }^{4}$, \\ Djamel Sayad $^{5}$, Said Mosbah ${ }^{1}$, J Rodreguez ${ }^{4}$, Yasir AlYasir ${ }^{2}$ and Raed Abd-Alhameed ${ }^{2}$ \\ \{czebiri@univ-setif.dz¹,wfaamshw@bradford.ac.uk, jsmkosha@bradford.ac.uk² \\ ${ }^{1}$ Department of Electronics, University of Ferhat Abbas, Sétif -1-, 19000 Sétif, Algeria. \\ ${ }^{2}$ School of Electrical Engineering and Computer Science, University of Bradford, BD71DP, UK. \\ ${ }^{3}$ Department of Electronics, University of Larbi Ben M'hidi, 4000 Oum-elbouagui, Algeria. \\ ${ }^{4}$ Instituto de Telecomunicações, Campus Universitário de Santiago, Aveiro, Portugal. \\ ${ }^{5}$ Department of Electrical Engineering, University of 20 Aout 1955-Skikda, 21000 Skikda, Algeria.
}

\begin{abstract}
In this work, we propose a metamaterial-based microwave sensor. The main body of the proposed sensor is a microstrip coupled complementary spiral rectangular resonator (CSRR). At resonance, a strong electric field is established along the sides of the CSRR creating a very sensitive area to change in the nearby of the dielectric environment. The enhanced proposed contactless sensor uses liquid samples placed normal to the sensor surface retained within capillary glass tubes. Quick analysis of the liquid dielectric properties is carried out by simply replacing the capillary tube with a new sample.The introduced liquid sample shifts the resonance frequency and alters the peak attenuation of the CSRR resonance. The liquid sample dielectric properties may be estimated by establishing an empirical relationship between the resonance characteristics and the liquid complex relative permittivity. In this work, we could improve our device by simple changing of the CSRR shape of a recently published work, this makes improvement on the characteristics of the sensor and lower its working frequency to $1.8 \mathrm{GHz}$.
\end{abstract}

Keywords: metamaterials, sensor, quality factor, bandwidth, resonant frequency, dielectric, characterization, complex permittivity. Spiral CSRR.

\section{Introduction}

Due to their low technological cost, their robustness and essentially their high sensitivity, microwave sensors have attracted considerable attention in many research domains such as: biomedical and chemical fields. Recently, a new type of sensors is being presented based on the concept of Metamaterials [1].

Metamaterials are electromagnetically considered artificial materials, they are based on resonators that can handle electromagnetic waves and bring new parameters and some unusual intrinsic properties that are not completely available in nature [2].

Metamaterials based structures are very important microwave components that are used in sensor designs. They are also used in a very wide frequency range, including microwaves [3-5], terra-hertz [6] and optics [7]. The type of substances that are tested on these sensors can be liquid solid dielectrics [7], and bimolecular [8]. Many studies on Metamaterials were performed 
to analyse the complex relative permittivity of the tested substances, where the dielectric constant and loss tangent could be retrieved [9].

The most used structures used in microwave sensors based on Metamaterials are usually Split Ring Resonators (SRRs) or Complementary Split Ring Resonators (CSRRs). These resonators may be manufactured with different shapes such as: micro-ribbon planar shapes [10] and thin films.

CSRRs and SRRs may have an electric field that is well defined along the Metamaterial structure, it is very sensitive close to the dielectric materials, and thus will produce variations in the resonant frequency and the resonator electric field Q-factor.

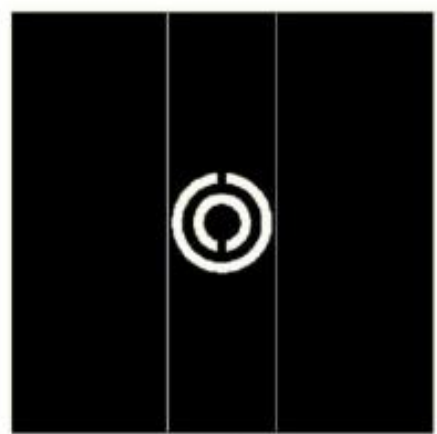

(a)

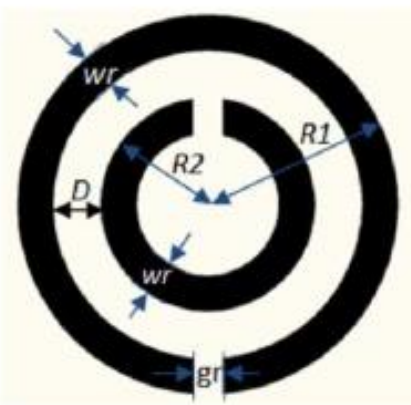

(b)

Fig. 1. Location of the CSRR resonator in the microstrip line [10].

The paper investigates on the optimization steps to design a new sensor based CSRR with a spiral shape. First of all, we simulate the CSRR sensor based on three rings as studied and realized in [11], so that we can compare and make comments on our results. Throughout the simulation of the structure, we were faced with a lot of problems such as:

1. Convergence problem: where three types of executions were carried out:Time Domain Solver, Frequency Domain Solver and Integral Equation Solver. The third type of resolution give us results that are very close to experimental ones, nonetheless at the expense of the execution time.

2. Execution problem: the execution type "Integral Equation Solver" requires a very important time compared to the other types, the computing time varies between $2 \mathrm{~h} 30 \mathrm{mn}$ for a single execution. This type of execution makes it possible to have a precise result at the expense of time.

3. Dimensions problem: we were faced with geometric problems related to the intersection of the rings by the CST software (CST). Based on these difficulties, we were obliged to choose a structure that converges quickly.

The sensor studied and realized in [11], was tested on a mixture of water andethanol. The motivation that makes the decision on testing ethanol comes from its high concentration and also for its wide use in our everyday life, such as: fuels, processes, medical and pharmaceuticals compositions. In such environments, the sensors must be potentially reliable with high sensitivity to measure accurately the ethanol concentration [11].

Among the novelties of this sensor structure, we mention the use of the electric field between the transmission line and the CSRR structure. The samples under test are placed on the 
horizontal plane of the sensor, which facilitates the rapid analysis of the intrinsic properties of liquid samples at microwave frequency band [11].

Another important characteristic of the proposed sensor is its contactless feature, in this case it can be used many times, in addition to being easy to use. It is also compact, economical and easy to manufacture. The sensor has shown the ability to successfully detect water in ethanol and to determine the water quantities validated by experimental measurements. The problem is that Chuma et al. [11] did not really validate these experimental results with simulation results, this is carried out by our prototype simulation.

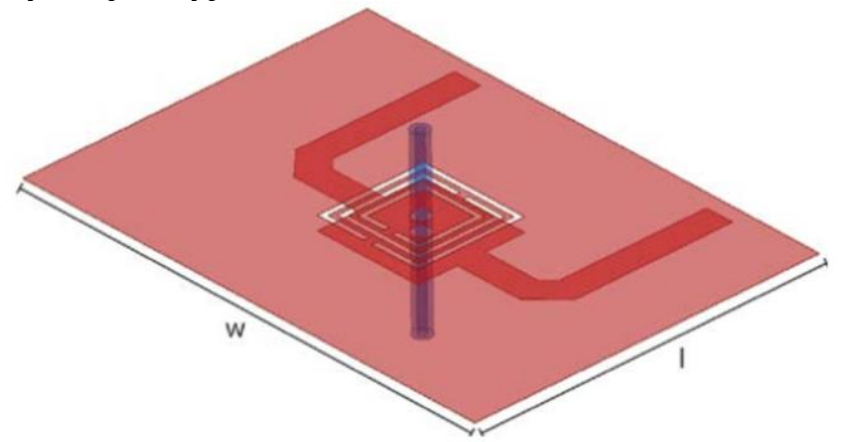

(a)

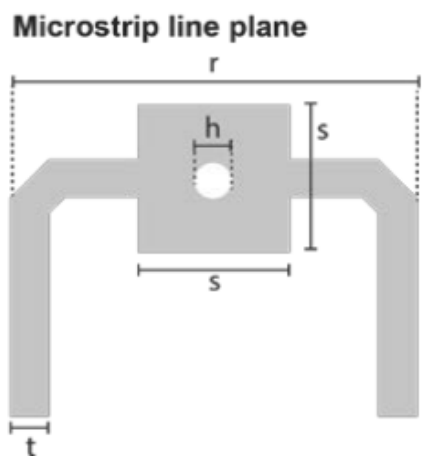

Ground plane

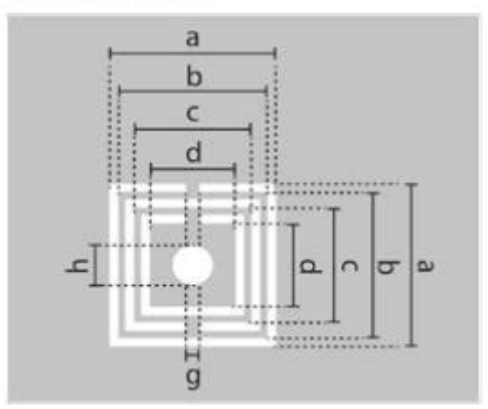

(b)
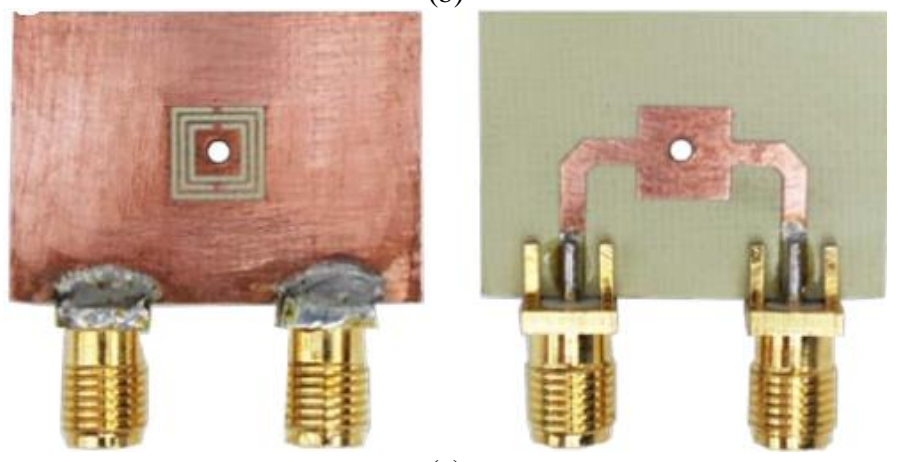

(c)

Fig. 2. (a) Sensor 3D view; (b) transmission line and CSRR dimensions: (c) Ground plane with CSRR (left) and transmission line (right) of the fabricated prototype. All dimensions are given in [11]. 


\section{Sensor Design}

Our study is inspired from the work presented in [11]. We carried out the simulation of the same structure so that we could validate our own sensor. The main constituents of the sensor proposed in [11], are the CSRR etched on the ground plane side and the transmission line printed on the other one. The hole is etched at the middle of the CSRR, the transmission line as well as the dielectric are simultaneously used to place the tube of the samples under test. Figure.2. a illustrates the 3D view of the sensor design, Figure2.b its geometrical dimensions and the realized prototype is shown in Figure2.c (the prototype CSRR sensor with a SMA connector).

The substrate used in the sensor design is Rogers RO3035 material with a thickness of 0.75 $\mathrm{mm}$, a relative permittivity $\varepsilon_{\mathrm{r}}=3.5$, and $\tan \delta=0.0015$. The capillary glass tubes are the commonly used tubes, clinically and laboratory, with a length of $75 \mathrm{~mm}$, an external diameter of $1.5 \mathrm{~mm}$ and an internal one of $1 \mathrm{~mm}$. The liquid solution used in the measurement is mixed with an ethanol solvent and distilled water.

In its pure form, the ethanol has a $9+9 \mathrm{j}$ complex relative permittivity at $1.9 \mathrm{GHz}$. For the water-ethanol mixture at different concentrations, the simulated or measured sensor transmission response thus allows the extraction of the resonant frequency and the $\mathrm{Q}$ factors. The extractions are used to solve the complex relative permittivity of the mixture of each pair of resonance frequency and the Q factor [11].

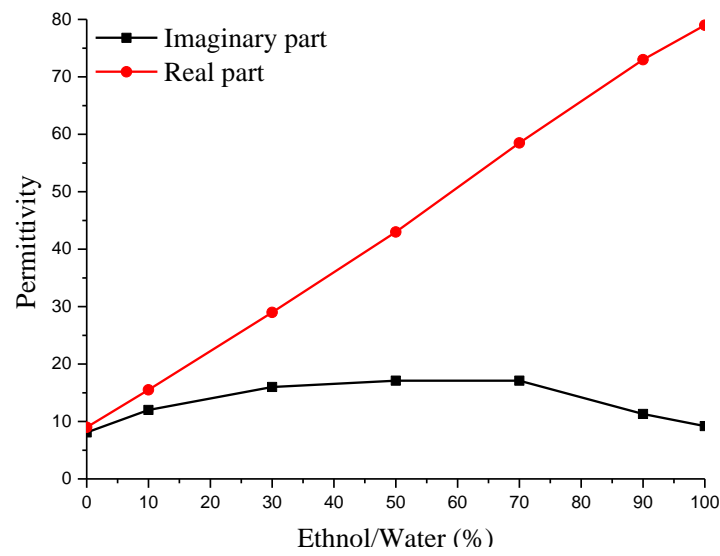

Fig. 3. Reference values of complex permittivities of water-ethanol mixtures [12].

Table 1. Reference values of water-ethanol mixture complex relative permittivities [12].

\begin{tabular}{|c|c|c|c|}
\hline water & Ethanol & $\bar{\varepsilon}$ & $\overline{\grave{\varepsilon}}$ \\
\hline $100 \%$ & $0 \%$ & 79.0 & 9.2 \\
\hline $90 \%$ & $10 \%$ & 73.0 & 11.3 \\
\hline $70 \%$ & $30 \%$ & 58.5 & 17.1 \\
\hline $50 \%$ & $50 \%$ & 43.0 & 17.1 \\
\hline $30 \%$ & $70 \%$ & 29.0 & 16.0 \\
\hline $10 \%$ & $90 \%$ & 15.5 & 12.0 \\
\hline $0 \%$ & $100 \%$ & 9.0 & 8.1 \\
\hline
\end{tabular}


Table I and Figure 3 present the reference values of er measured by Bao et al. in 1995 [12]. The microwave characterization of this mixture was carried out using a coaxial probe.

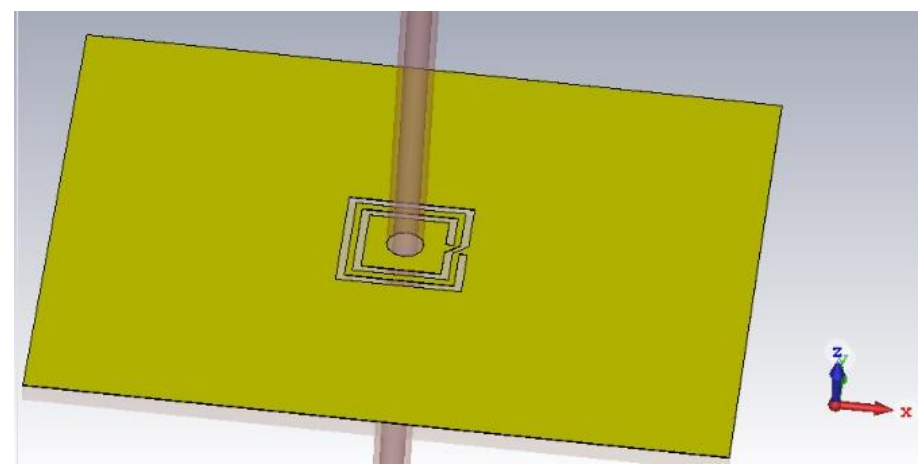

Fig. 4. Our structure similar to that of [12] in the CST interface.

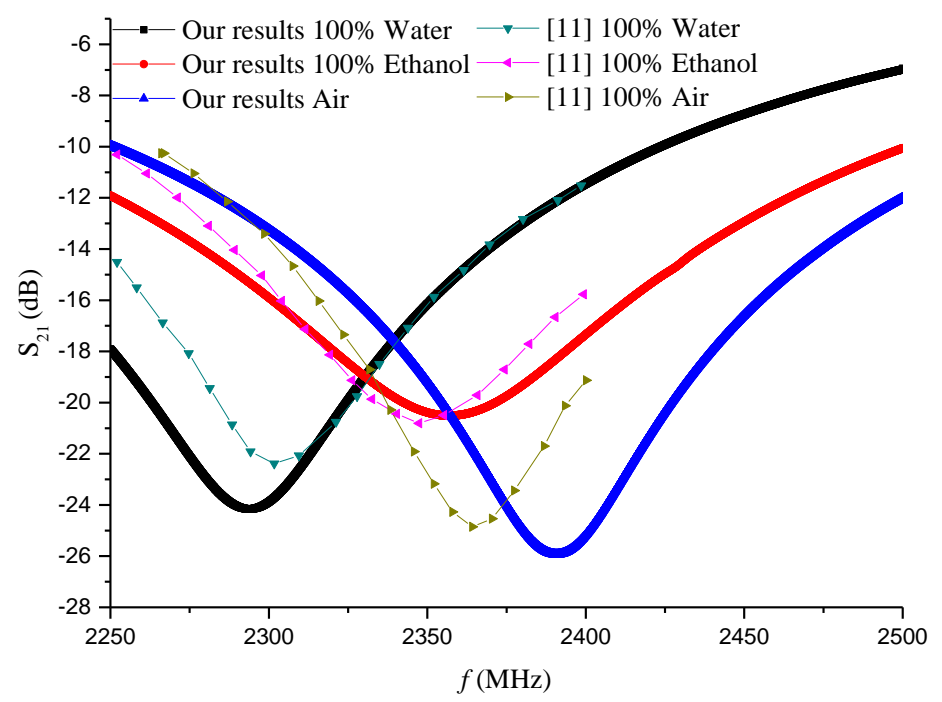

Fig. 5. Comparison of the simulated S21 response with that measured [11] of the water-ethanol mixture of the three different cases.

\subsection{Validation of the same structure realized by [11]}

Our structure simulated in CST software is shown in Figure 4. The transmission coefficients of the three cases (air, $100 \%$ water and $100 \%$ ethanol) simulated and their quality factors are compared with those measured $\left(S_{21}\right.$ and $\left.Q\right)$ in [11], as shown in Figures 5-7. A good concordance is observed, this allows us to validate our reshaped structure and our proposed structure. Misfortune, he did not validate his results with simulation software, so we managed to validate them with our simulation results obtained by CST.

Figures 3, 4, 5, 6 and 7 show that the resonant frequencies decrease by increasing the proportion of ethanol, whereas for the case of the quality factor $\mathrm{Q}$, we notice that this one 
increases with the increase of the proportion of ethanol in water. This difference is consistent with results obtained through numerical and simulation methods found in other recently published works [11].

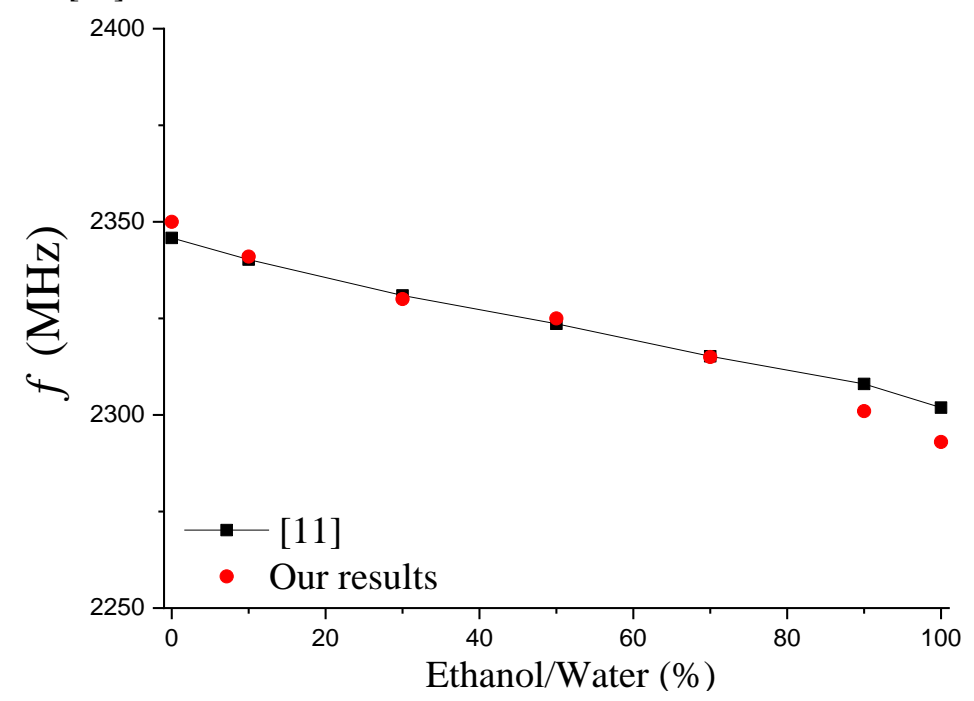

Fig. 6. Comparison of the resonance frequency extracted from the simulated results with that of [11] of the water-ethanol mixture for two cases (100\% water - $100 \%$ ethanol).

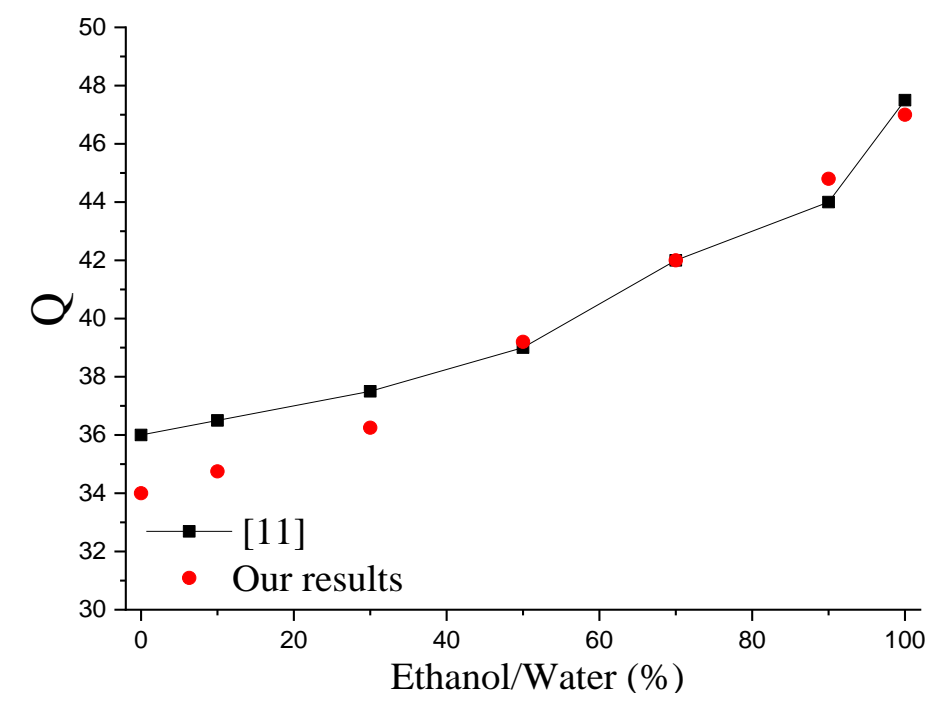

Fig. 7. Comparison of the quality factor extracted from the simulated results with those of [11] of the water-ethanol mixture for two cases (100\% water - $100 \%$ ethanol). 


\section{Our Proposed Structure}

Our simulated structure is shown in Fig. 8, where the changes made were essentially based on the resonator shape, while preserving the other dimensions and the sensor design studied in $[1]$.

The dimensions as well as the medium used are described in Fig. 2 with the substrate. Except that the link between two rings has been added, this has allowed us to completely change the CSRR to a spiral one.

When the transmission line is powered by a microwave signal, the spiral CSRR is excited by the induced voltage difference between the capacitive CSRR plate and the ground plane. Therefore, a resonance occurs, during this resonance and an electric field is created in the space between the CSRR capacitive plate and the square resonator. This makes the internal and close region of the CSRR sensitive to dielectric changes. Hence, this region inside the CSRR may be used to measure the dielectric intrinsic properties.
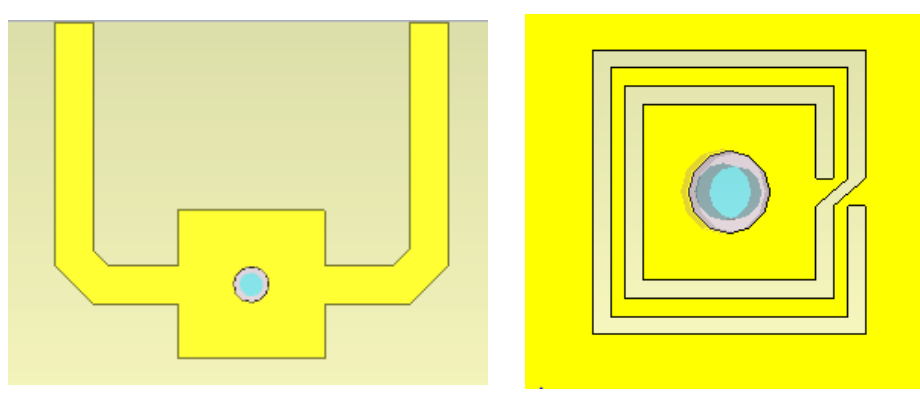

Fig. 8. The proposed sensor: transmission line (left) and ground plane with spiral CSRR (right)
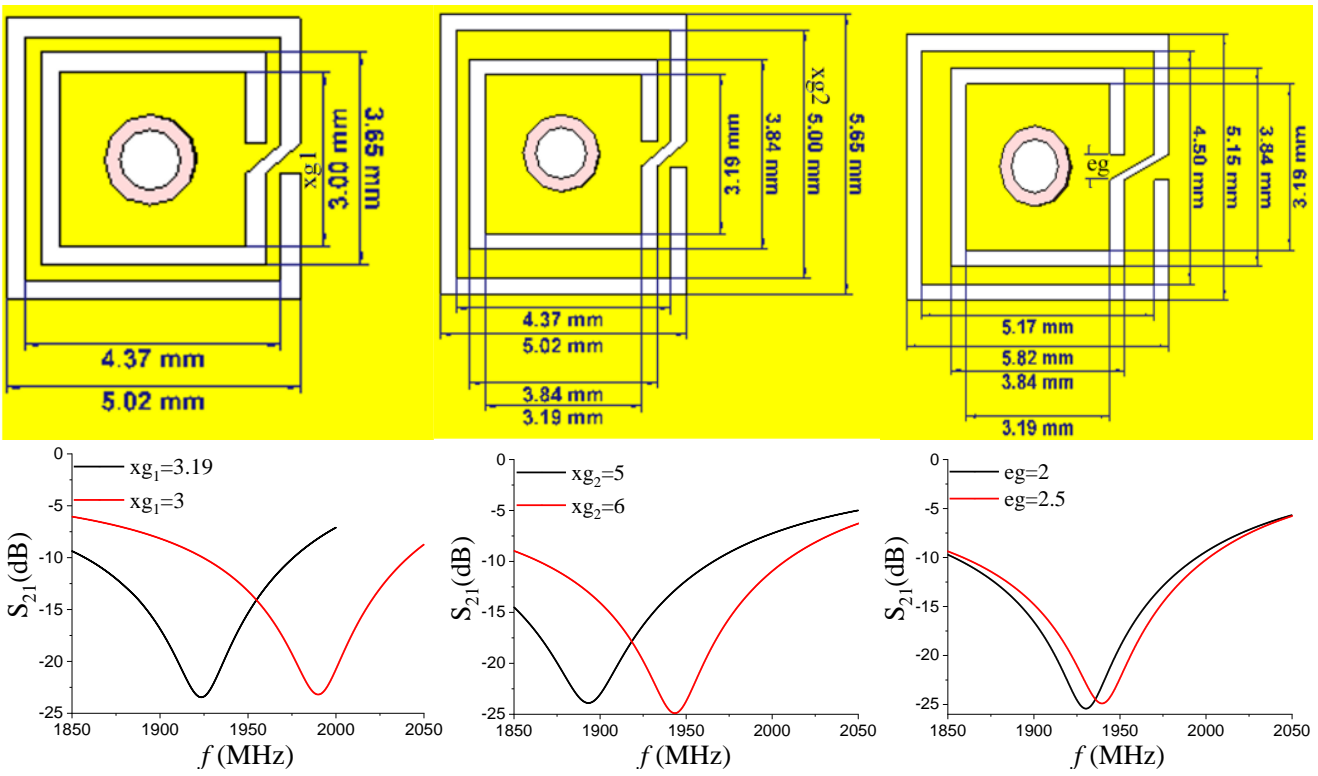

Fig. 9. Changes made to our Spiral CSRR level 


\subsection{Parametric study}

In order to design an optimized sensor, we started this work with a parametric study. First, we studied the influence of the different geometrical parameters $\left(\mathrm{eg}_{1}, \mathrm{xg}_{1}\right.$ and $\left.\mathrm{xg}_{2}\right)$ of the resonator on the resonant frequency and the parameter S21 that characterize the sensor. For this reason, certain geometrical parameters of the resonator will be modified. According to Figs. 9, the optimum values of the geometrical parameters $\mathrm{e}_{1}, \mathrm{~g}_{1}$ and $\mathrm{g}_{2}$ are $0.265,3.19$ and $4.5 \mathrm{~mm}$, respectively.

\subsection{Evaluation of the microwave sensor parameters}

Figures 10, 11and 12 show the effect of vertical displacement of the membrane on the resonant frequency and the variation of the sensitivity of the three sensors respectively.

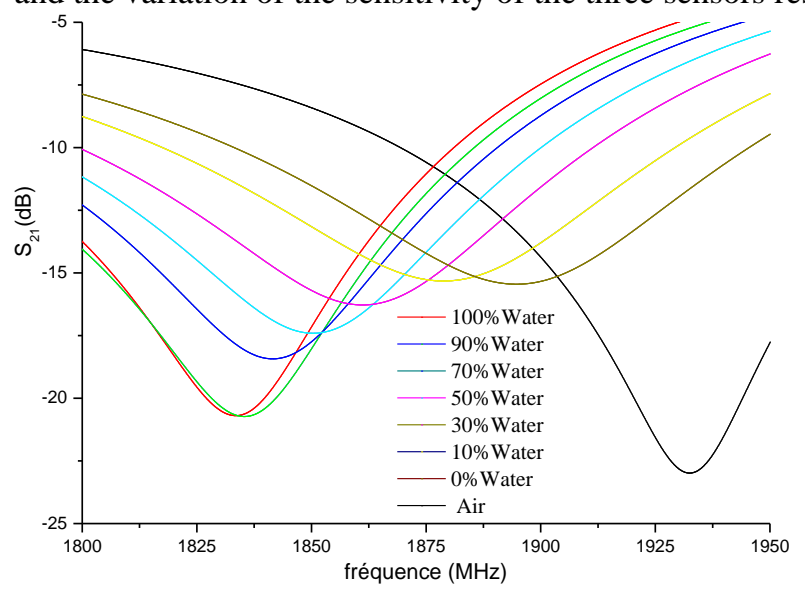

Fig. 10. The simulated $\mathrm{S}_{21}$ response of our proposed sensor according to the water-ethanol concentration

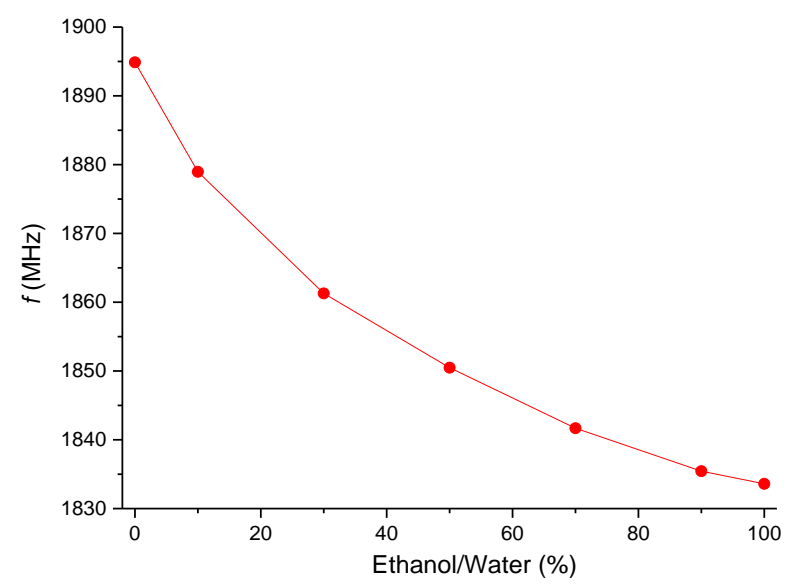

Fig. 11. Resonance frequency extracted from the simulated results of our new sensor. 


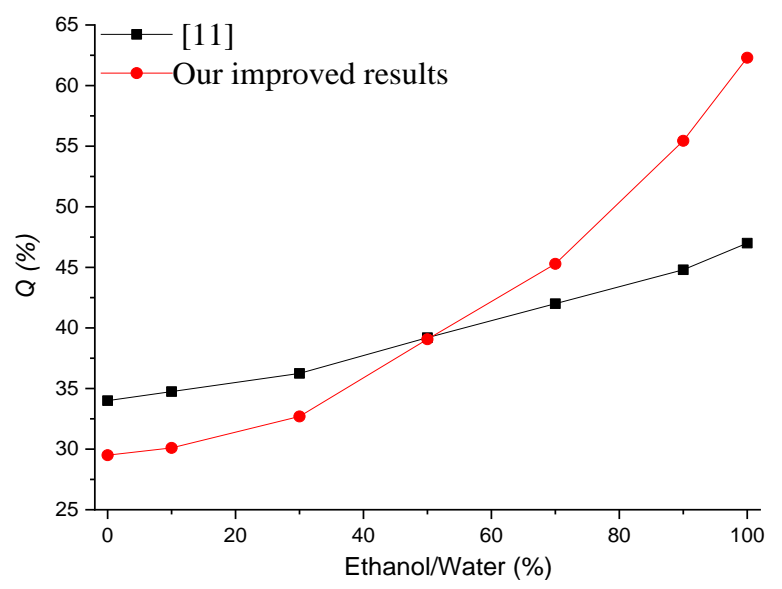

Fig. 12. Quality factor extracted from the simulated results of our new sensor.

The simulation results of the transmission coefficient S21, the resonant frequency, and the quality factor Q for the eight tests carried out on different samples are shown in Figures 10-12. It can be clearly seen that, the resonant frequency goes from $1.830 \mathrm{GHz}$ to $1.950 \mathrm{GHz}$ when the volume ratio of the water goes up from $10 \%$ to $90 \%$. The minimal attenuation occurs at a water volume ratio of $10 \%$. It should be noted that the non-linearity seen in the maximum attenuation with respect to the water content follows the non-linear loss function of the water mixture. Thus, we were able to improve the structure proposed by Chuma et al. in [11], for high concentrations of more than $50 \%$ and we were able to minimize the sensor's working frequency from $2.4 \mathrm{GHz}$ to $1.86 \mathrm{GHz}$.

\section{Conclusion}

A microwave CSRR-based sensor operating in the high frequency range around $1.86 \mathrm{GHz}$ is proposed. It consists of a common capillary glass tube inside which the liquid samples under test are placed. The electromagnetic interaction between the resonator, the transmission line and the capillary tube containing the sample (test) is translated by the better sensitivity of our improved device, and the simplicity of this device lies in the possibility of changing the capillary tube quickly and easily. It is therefore a good choice for the measurement environment.

In terms of proof of concept, an empirical model will be established in future works to retrieve the complex dielectric intrinsic properties of water-ethanol liquid mixture samples from the measured resonant frequency and the $\mathrm{Q}$ factor. Therefore, it would be possible to determine the fraction quantity of water or ethanol in the water-ethanol mixture.

\section{References}

[1] Al-Naib, I. (2016). Biomedical sensing with conductively coupled terahertz metamaterial resonators. IEEE Journal of Selected Topics in Quantum Electronics, 23(4), 1-5.

[2] Ziolkowski, R. W., \& Engheta, N. (2006). Introduction, history, and selected topics in fundamental theories of metamaterials. Metamaterials: Physics and Engineering Explorations, 1-41 
[3] Zebiri, C., Lashab, M., Benabdelaziz, F., Abd-Alhameed, R. A., \& Elmegri, F. (2015). A Grating Monopole Antenna on Metamaterial Using MSRR for DVB-T Application. In Applied Mechanics and Materials (Vol. 789, pp. 989-992). Trans Tech Publications.

[4] Lashab, M., Zebiri, C. E., Djouablia, L., Belattar, M., Saleh, A., Benabdelaziz, F., \& AbdAlhameed, R. (2018). Characterization of horn antenna loaded with CLL unit cell. Microwave and Optical Technology Letters, 60(8), 1847-1856.

[5] Lashab, M., Jan, N. A., Benbdelaziz, F., \& Zebiri, C. E. (2018). Electrically Small Planar Antennas Based on Metamaterial. In Antenna Fundamentals for Legacy Mobile Applications and Beyond (pp. 71-98). Springer, Cham.

[6] Su, L., Mata-Contreras, J., Vélez, P., Fernández-Prieto, A., \& Martín, F. (2018). Analytical method to estimate the complex permittivity of oil samples. Sensors, 18(4), 984. [7] Li, J. (2017). Permittivity measurement of low-loss substrates based on split ring resonators. World Journal of Engineering and Technology, 5(04), 62.

[8] Lee, H. J., \& Yook, J. G. (2008). Biosensing using split-ring resonators at microwave regime. Applied Physics Letters, 92(25), 254103.

[9] Ebrahimi, A., Withayachumnankul, W., Al-Sarawi, S., \& Abbott, D. (2013). Highsensitivity metamaterial-inspired sensor for microfluidic dielectric characterization. IEEE Sensors Journal, 14(5), 1345-1351.

[10] Alibakhshi-Kenari, M., Naser-Moghadasi, M., \& Sadeghzadeh, R. A. (2015). Bandwidth and radiation specifications enhancement of monopole antennas loaded with split ring resonators. IET Microwaves, Antennas \& Propagation, 9(14), 1487-1496.

[11] Chuma, E. L., Iano, Y., Fontgalland, G., \& Roger, L. L. B. (2018). Microwave sensor for liquid dielectric characterization based on metamaterial complementary split ring resonator. IEEE Sensors Journal, 18(24), 9978-9983.

[12] Withayachumnankul, W., Jaruwongrungsee, K., Tuantranont, A., Fumeaux, C., \& Abbott, D. (2013). Metamaterial-based microfluidic sensor for dielectric characterization. Sensors and Actuators A: Physical, 189, 233-237. 